\title{
Exploration in Features and Skills of Scientific English Translation
}

\author{
Wang Yuehui ${ }^{1, a}$ \\ ${ }^{1}$ Collage of foreign languages, Dalian jiaotong university, dalian, liaoning, 116021,China \\ awwtwyh@163.com
}

Keywords: Scientific English translation; features; skills.

\begin{abstract}
Problems such as large vocabulary, very professional, and distinct cultural difference, exist in scientific English translation; therefore, it is very difficult and important to translate scientific English in an exact way; this paper starts from the features scientific English translation, and explores the ways to make scientific English translation exact and smooth and the means to optimize in a deeper level in combination with cultural background, aiming to provide some advice for translators of scientific English.
\end{abstract}

\section{Introduction}

As a professional skill, scientific English translation is faced with more complicated forms and content than daily scientific oral and written English, needs higher requirements in the expressions of translation language, and meets professional vocabulary or techniques frequently, under which circumstance translators have to refer to or learn related background knowledge and understand the cultural background and special expressions of western countries. Thus, compared with ordinary scientific English expressions, scientific English translation has higher professional requirements and the goal of translation is to transform the ideas expressed by one language into another form to satisfy the reading or understanding needs of another language background through lingual explanation.

\section{Features of Scientific English Translation}

Scientific style advocates rigorousness, exactness, logic, and conciseness, with outstanding highlights, neat sentences and little changes, and preposed expressions are often used, which means that main information is preposed in the sentence and the subject delivers main information.

The features of scientific English translation are to be neat, exact, concise, and rigorous. Then, how to deal with the language structure of scientific articles is a problem to be explored in English-Chinese Scientific translation. For the translators of scientific English, it is a professional job, difficulties and similarities exist in the process of translation, which can be concluded as the following points.

\section{Difficulty in understanding professional expressions}

Scientific terms refer to the expressions in science referring to certain professional concepts. Therefore, it should be noticed in translation process and cannot be ignored. Scientific terms are professional with many meanings; so they should be dealt with according to professional content in translation process, otherwise, great errors may happen with a little carelessness. For example, some people mistranslate "the newly developed picture tub" into "lately image tub is developed", and some other translator mistranslated "a unique instant-picture system” into "unique image system”, and so on. Here is another example: the term "cassette", except for other meanings, as audio tape, has two meanings, "packing tape box" and "case style tape", and the meaning should be decided according to the context. For example, in the specifications of imported radio recorder, there are two subtitles: "checking the cassette" and "to insert cassette", in which the meanings are different; the first cassette means the tape and the second means the box. For translators, the the difficulty of translation from English to Chinese of scientific articles is no less than the production of the original text of scientific 
English; some professional or special vocabulary may need great energy and cost to be appropriate. There are many differences and changes in the expressions of Chinese and English, and the translation should not only correspond to the original text in expressions, but also be proper in the place and times of expressions, to be smooth and exact. Professional scientific English translation should be able to be faced with numerous professional environment, where many words have more than one meaning and present in abbreviations, and therefore, it is hard to be referred in ordinary dictionary; so it is very important to translate these expressions exactly. However, some words seem ordinary in form, but they have different meanings in particular professional environment, which requires translators to know deeper about professional environment.

\section{Difficulty in the integration of cultural differences}

Every race around the world has its unique cultural connotation, and therefore, strong cultural features show in the lingual expressions; language is a special carrier in cultural field, which can be conveyed and passed down through language and can be influenced by culture; thus, language contains different expressions, thoughts and living styles besides the basic cultural connotation. Translation is a kind of communication across cultures, and there are great differences between Chinese and Western cultures, which leads to obstacles in communication, and translators are needed to understand the mechanisms of two kinds of cultures, to do best to solve the paradoxes and conflicts in cultural differences, at the same time of respecting the other culture and helping their native people to understand the other culture. If translators only pay attention to the exactness of words, mistranslation may happen, and mature translators should be able to understand the meaning both on lingual level and cultural level; otherwise, translation is just literal, and cannot be exact and perfect in deep level.

\section{Skills in Scientific English Translation}

Since the reform and opening-up, our country has introduced and digested large amount of advanced techniques and methods from abroad under the idea of "the experience of the others can help solve our own problems". Against this kind of large environment, the translation of scientific English becomes the key and base of technique introduction and digestion. English and Chinese originate respectively from the western alphabetic writing and oriental pictograph, and large differences exist in grammar, cultural backgrounds and language customs. The interrelations and differences should be carefully analyzed, so as to find out the corresponding laws between English and Chinese in scientific literature and express the key content in science and technology. One of the features of scientific literature is to use scientific terms and vocabulary. Scientific terms have strong information function and are the starting point and basis for scientific study and communication. The importance and necessity in translation and related studies of scientific term becomes more and more outstanding, and the whole process of translation experiences three procedures of understanding original text, transforming languages and proofreading and modification, the three of which can not be ignored; the translation should be guided under scientific and overall techniques and follow objective regulations, only in this way can the original text and translating methods in front of different readers be analyzed.

\section{Grasp the exactness of vocabulary context}

The meaning of certain word should be defined before understanding its class. Many words belong to different classes in different sentences, and different word classes may lead to different meanings, which is also homonymy phenomenon. Under this circumstance, we should define meanings according to the world class in sentences. For example, We name all PCs which are fitted with acertain amount of workspace memory RAM. What needs to be mentioned is that all the computers have some memory workplace which can be called as RAM. Many words have total different meanings in ordinary English and scientific English, as well as in different majors. In the translating 
process, meanings of words should be defined according to the content of majors. During translating process, sometimes it is hard to find proper meaning in English dictionary, in which case, if one translates word-for-word randomly, the translated articles would be stiff and obscure and cannot express the original meaning exactly, which may cause misunderstanding. At this time, translators should start from the basic meaning of this word and extend the meaning on the basis of the context content and logical relationship. Scientific English translation often face complex professional knowledge in professional fields, which needs translators to know the related content in this aspect and master the core meaning in the original context so as to understand in an exact way. Especially when the sentence is too long or there are too many subordinated clauses, translators should understand the lingual expressing structure more clearly and analyze the logical relationship deeply, with many translating methods to transfer language expressing forms, so as to avoid word-for-word translation; in this way, the original text can be understood and personal potential can be fully developed.

\section{Grasp the tones and tenses of the original text}

The first principle of translation is to respect the accuracy of original text, which cannot be tampered, and the style of original text should also be expressed well, for example, the style of the period and authors' linguistic features should be kept. For example, when the sentence "I am up to my neck in your bullshit." is translated, the literal meaning is "you are destroying me”, but if it can be noticed that this is said by a soldier in a movie, this can be translated as "you bring unluckiness to me." Both respect and smoothness should be taken into consideration in translation; scientific English translation should not only express professional content, but also be smooth and fluent, making readers rapidly understand the professional knowledge or language situation. For example, when the sentence "It is then passed to a purification system for treatment and from there to the clean heavy fuel tank, which is known as the service tank." Is translated, there is a infinitive form, a subordinate clause and a comma; as in Chinese, the custom is to put attribute forward, the original sentence can be transformed into two coordinate sentences, for example: "The fuel is sent to a purification system for treatment, and then to the clean heavy fuel tank; the fuel tank is known as the service tank.”

\section{Grasp the connotation of cultural differences}

Culture can transmit information and feelings in the language communication among people; besides the basic words and language background, translators should also have cultural awareness, especially when the proverbs, mottos, allusions and other special linguistic forms appear, translators should clearly know the geographic position and other knowledge such as culture, history and religion. Scientific English originates from Britain; as it is surrounded by the sea, to increase the contact with the outer world, navigation career was developed, and thus, much vocabulary is related to navigation in its lingual background. For example, the meaning of phrase "trim the sail to the wind" means to take advantage of an opportunity. Thus, in translation, translators should learn the background of language source and the implication in particular settings; it is necessary to keep sensitive and alert towards cultural differences.

\section{Examine the mistakes and omissions in target language}

As the last step of translation, examination is essential, which is to optimize the previous translation and deliberate the implication and words of the original text further; what should be noticed is that language should be smooth and standard, the punctuation should be exact, words and sentences should not be omitted, great expressing errors caused by deficiency of certain words should be avoided. Therefore, strong sense of cultural responsibility and meticulous attitude are needed in translation; even though it is the last procedure, repetition is needed, and after the completion of the whole translation, it is necessary to invited other people to read through, to put forward some advice 
on modification and to ensure the smoothness and coherence of the whole text and the exactness of expressions, so as to be perfect in translation.

\section{Conclusion}

Generally speaking, scientific English translation is a kind of language activity to transform English into other language forms; it seems like a kind of literature work on the surface, but it shows the understanding and communication on a deeper level; therefore, to realize smooth and exact translation of scientific English, translators should fully understand and think over the real meaning of the words in both original and translated versions, to try one's best to make special language expression against its own cultural features on the basis of respecting the source text, for the communication and inheritance is often produced in the translating process. The particularity of scientific English makes the translation different from the ordinary English; the word formation characteristics of scientific English should be grasped, and at the same time, the popularity of scientific English causes differences among different majors; in translating process, the basic content and background of original text should be understood; only in this way, can the exactness and rationality of translation be gained.

\section{References}

[1] Zeng Zhaotao. On Pragmatic Translation Theory of Higher Vocational Education [J] Shanghai: Shanghai Journal of Translators, 2008 (1): 49-52.

[2] Lv Ling. Brief Analysis on Learning Methods of Foreign Language Translation of Higher Vocational Education. [J]. Beijing: Labor Union Expo: Theoretical Research, 2011(1): 130.

[3] Zhang Hongsheng: Brief Discussion on Skills of Scientific English Translation [J]. Beijing: Beijing Electric Power Junior College Journal, 2010， 29(3):8-9.

[4] Chen Lanying, Tan Yumei. Teaching Problems and Countermeasures of Basic Scientific English Course Translation of Higher Vocational Education. [J]. Fuqing: Fujian Normal University Fuqing Branch School Journal, 2008(3):65-68.

[5] Gong Xuan. Problems and Countermeasures of Scientific English Translation Teaching of Higher Vocational Education. [J]. Nanchang: Education Research Monthly, 2011(12):100-101. 